\title{
Generation and decay of the magnetic field in collisionless shocks
}

\author{
Mikhail Garasev and Evgeny Derishev \\ Institute of Applied Physics of the Russian Academy of Sciences, \\ 46 Ulyanova Str., Nizhny Novgorod 603950, Russia \\ email: garasev@appl.sci-nnov.ru
}

\begin{abstract}
We present the results of numerical particle-in-cell (PIC) simulations of the magnetic field generation and decay in the upstream of collisionless shocks. We use the model, where the magnetic field in the incoming flow is generated by continuous injection of anisotropic electronpositron pairs. We found that the continuous injection of anisotropic plasma in the upstream of the shock-wave generates the large-scale, slowly decaying magnetic field that is later amplified during the passage of the shock front. In our simulations the magnetic field energy reached $\sim 0.01$ of the equipartition value, after that it slowly decays on the time scale proportional to the duration of the injection in the upstream. Thus, the magnetic field survives for a sufficiently long time, and supports efficient synchrotron radiation from relativistic shocks, e.g., in GRBs.
\end{abstract}

Keywords. shock waves, magnetic fields, gamma-ray burst

\section{Introduction}

Relativistic collisionless shocks are common phenomena in extreme astrophysical sources like AGNs and GRBs. Such shocks are thought to be capable of generating strong magnetic fields and particle acceleration (Achterberg et al. (2001), Gruzinov (2001), Medvedev \& Loeb (1999)). This makes them natural sources of synchrotron emission. These theoretical predictions have been tested by various numerical simulations (Spitkovsky (2008), Keshet et al. (2009), Sironi et al. (2013), Sironi et al. (2015)).

However, these numerical simulations have discovered some problems in this simple picture. It was found that the magnetic field generated at the shock front via Weibel instability decays very fast on the time scale comparable to the inverse plasma frequency. If the strong magnetic field initially exists in the upstream, then no particle acceleration is observed. So, strong magnetic fields and particle acceleration never meet each other, leaving no room for efficient synchrotron emission.

Solution to this problem could be linked with presence of magnetic turbulence in the upstream flow (Medvedev \& Zakutnyaya (2009), Miloslavljevic \& Nakar (2006)). Recently a new model of the relativistic collisionless shock was proposed (Derishev \& Piran (2016)), where the magnetic field growth is a two-stage process. In the first stage, the magnetic field is generated upstream of the shock as a result of massive production of electron-positron pairs with anisotropic velocity distribution in the fluid comoving frame. The width of this region is about the electron cooling length, which is many orders of magnitude greater than the plasma skin depth, so that the magnetic field is growing very slowly and could be maintained for a very long period of time compared to the plasma time scale. In the second stage, this magnetic field is enhanced at the shock front, and then decays in the downstream. According to the model, the decay length is given by the spatial scale of the turbulent magnetic field formed at the long stage of initial build-up. The goal of this model is to achieve the magnetic field decay length comparable to the electron cooling length. 
In this paper we present results of PIC simulations of the magnetic field generation in the upstream of collisionless shocks. The magnetic field build-up results from slowly developing Weibel instability in initially isotropic pair plasma with gradual injection of anisotropic component via generation of electron-positron pairs in collisions of highenergy photons escaping from the shock. In our simulations, we follow the evolution of a small (and, therefore, uniform) region in the comoving frame. Simulations start in the upstream with constant injection rate for anisotropic $e^{+} e^{-}$pairs, passes through a phase of the magnetic field growth, the rate of which depends on the rate of injection, and ends with the observation of the magnetic field decay.

\section{Overview of simulation setup}

For our simulations we use relativistic PIC-code EPOCH (Arber et al. (2015)) in Cartesian 2D geometry ( $\mathrm{x}$ - and $\mathrm{y}$ - axis are in the simulation box, $\mathrm{z}$-axis perpendicular to the simulation box) with periodic boundary conditions. The code was modified to allow injection of new electron-positron pairs (Garasev \& Derishev (2016)). During the injection step we randomly distribute particles across the computational domain ensuring that there is no perturbation to the total momenta, charge or current. For this purpose we simultaneously inject two electron-positron pairs with equal but oppositely directed velocities. We take 1000 particles per cell per species in each simulation in order to reduce the numerical noise. The low noise levels are necessary to explore the decay of the magnetic field on long time scales.

Initially our simulation box was filled with isotropic Maxwellian $e^{+} e^{-}$plasma whose distribution function is

$$
f_{e, e^{+}}(p)=\frac{N_{0}}{\sqrt{(2 m e T)^{3}}} \exp \left(-\frac{p_{x}^{2}}{2 m_{e} T_{x}}-\frac{p_{y}^{2}}{2 m_{e} T_{y}}-\frac{p_{z}^{2}}{2 m_{e} T_{z}}\right),
$$

where $m_{e}$ is the electron mass, $T_{x, y, z}$ the temperatures measured in the energy units, $p$ the momentum of a particle and $N_{0}$ the number densities of electrons and positrons. The initial temperature was set to $T_{x, y, z}=50 \mathrm{keV}$. We used Cartesian 2D grids with uniform mesh with $1600 \times 1600$ cells. The size of a cell in each direction was set to $r_{D}=\sqrt{k T /\left(8 \pi e 2 N_{0}\right)}$. Here $e$ is the elementary charge. Injection of anisotropic pairs starts at the beginning of each simulation at the moment $t_{i}<0$, continues with a fixed rate, and then stops at $t=0$. The injected plasma has elongated two-temperature distribution function with $T_{x}=200 \mathrm{keV}$ and $T_{y, z}=50 \mathrm{keV}$. The total number density of the injected component is $\delta$ times the initial number density of particles. We track the evolution of a magnetic field for up to $15000 / \omega_{\mathrm{p} 0}$, where

$$
\omega_{\mathrm{p}}(t)=\sqrt{8 \pi e^{2}\left(N_{0}+N_{\mathrm{a}}(t)\right) / m_{\mathrm{e}}}
$$

is the plasma frequency, $\omega_{\mathrm{p} 0}=\omega_{\mathrm{p}}\left(-t_{\mathrm{i}}\right)$ the background plasma frequency and $N_{\mathrm{a}}(t)$ is the number density of injected component. It is convenient to use dimensionless time

$$
\tau=\int_{-t_{\mathrm{i}}}^{t} \omega_{\mathrm{p}} \mathrm{d} t-\tau_{\mathrm{i}}, \quad \tau_{\mathrm{i}}=\int_{-t_{\mathrm{i}}}^{0} \omega_{\mathrm{p}} \mathrm{d} t
$$

\section{Results}

We performed a number of simulations with different injection durations. In agreement with the standard picture of the Weibel instability, the magnetic field filaments start to 


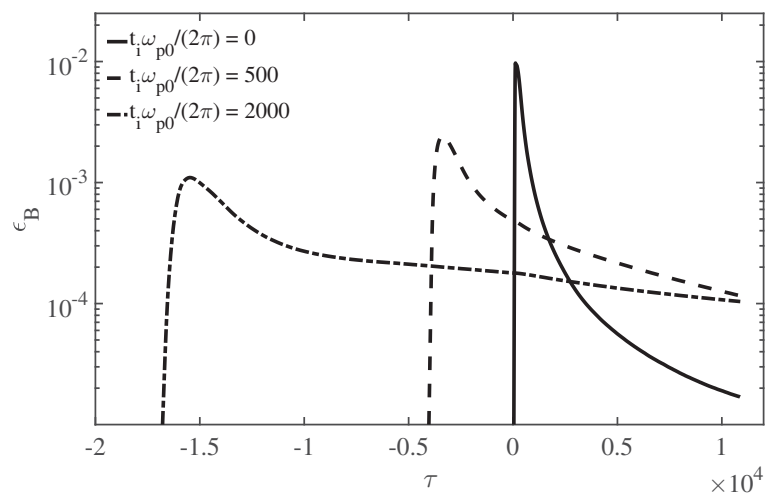

Figure 1. Magnetization parameter (the ratio of the magnetic energy density to the particles energy density) $\epsilon_{B}$ over time for different injections: instantaneous (solid line), $\omega_{\mathrm{p} 0} t_{\mathrm{i}} /(2 \pi)=500$ (dashed line), and $\omega_{\mathrm{p} 0} t_{\mathrm{i}} /(2 \pi)=2000$ (dash-dotted line). In all simulations $\delta=2$.

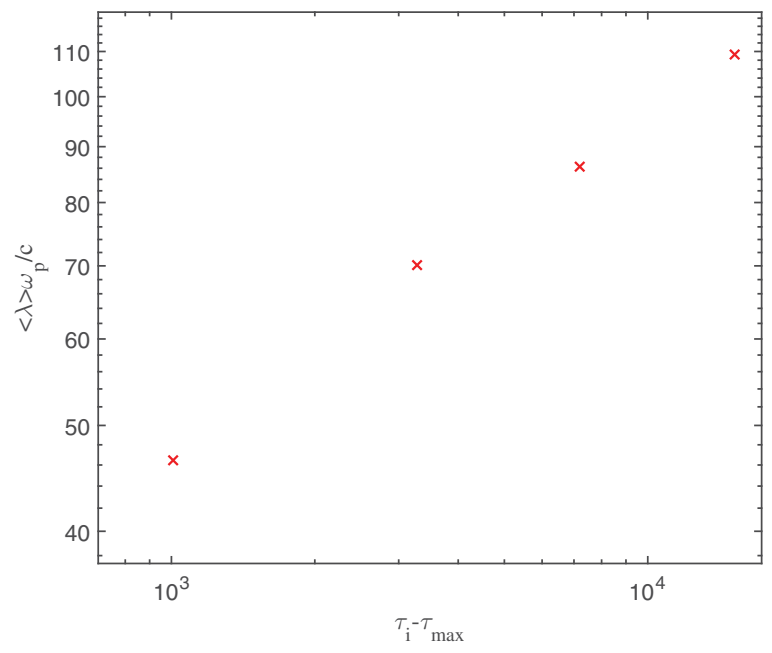

Figure 2. The average wavelength of magnetic field filaments $\langle\lambda\rangle$ at the end of the injection phase as a function of $\tau_{\mathrm{i}}-\tau_{\max }$, where $\tau_{\max }$ is the time of the maximum magnetic field energy.

grow with relatively small spatial scales. The fastest growing modes have their wave vectors nearly perpendicular to the axis of anisotropy. The growing magnetic field deflects the particles and smears out the anisotropy, eventually stopping the magnetic field growth. After that the magnetic filed starts to decay. The decay rate is largest for the short wavelengths. Thus, in the decay phase the spatial scale of the magnetic turbulence grows.

In Fig. 1 we plot three examples of the magnetic field evolution with different injection duration (as observed in our simulations). The maximum value of the observed peak magnetization is about 0.01 . This relatively small value is probably due to the small anisotropy of injected component used in our model. It is clearly seen, that the longer injection time corresponds to lower peak magnetization and to longer decay time. As a result, after certain time, the magnetic field generated by long injection overcomes the magnetic field generated by short injection. We attribute this to a larger spatial scale of a magnetic field at the moment of maximum magnetization and to subsequent washing-out of modes with short wavelengths during the rest of injection period (See Fig. 2). From 


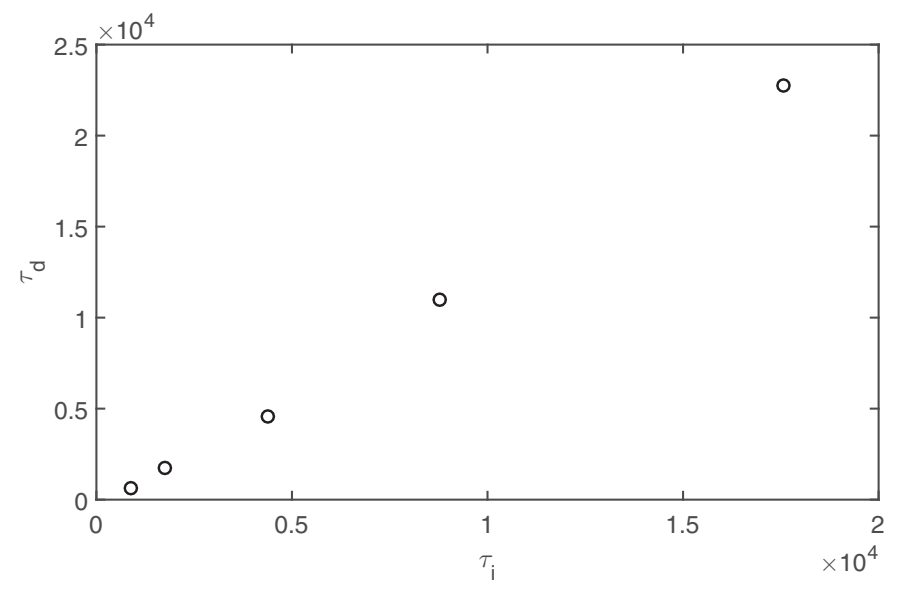

Figure 3. Dissipation timescale $\tau_{d}$ of a magnetic field (it is defined as $\tau_{\mathrm{d}}=\left(\tau_{2}-\tau_{1}\right) / \ln \left(\epsilon_{\mathrm{B}}\left(\tau_{1}\right) / \epsilon_{\mathrm{B}}\left(\tau_{2}\right)\right)$, where $\tau_{1}=0.1 \tau_{\mathrm{i}}$ and $\tau_{2}=0.25 \tau_{\mathrm{i}}$ are two moments of time after injection has stopped) as a function of the injection time.

the simulations we found that the typical spatial scale of the generated magnetic field is much larger than the plasma skin-depth $c / \omega_{\mathrm{p}}$ and increases with injection time as

$$
\langle\lambda\rangle \propto\left(\tau_{\mathrm{i}}-\tau_{\max }\right)^{1 / 3} .
$$

In Fig. 3 we present the measured dependence of the magnetic field dissipation time as a function of the injection time. We observed that the field dissipation time approximately equals to the injection duration a least up to $t_{i}=20000 \omega_{\mathrm{p}}^{-1}$.

We show that the prolonged injection leads to build-up of relatively large-scale magnetic field in the upstream due to development of the Weibel instability. Moreover, this large-scale magnetic field could be amplified at the shock front preserving its scale and then could survive for a long time in the downstream, explaining efficient synchrotron emission from relativistic shocks, e.g. in GRBs.

This work was supported by the Russian Science Foundation grant No 16-12-10528.

\section{References}

Achterberg, A., Gallant, Y. A., Kirk, J. G., \& Guthmann, A. W. 2001, MNRAS, 328, 393

Gruzinov, A. 2001, Astrophys. Jour. Lett., 563, L15

Medvedev, M. V. \& Loeb, A., 1999, ApJ, 526, 697

Spitkovsky, A. 2008 Astrophys. Jour. Lett., 682, L5

Keshet, U., Katz, B., Spitkovsky, A., \& Waxman, E., 2009, ApJ, 693, L127

Sironi, L., Spitkovsky, A., \& Arons, J., 2013, ApJ, 771, 54

Sironi L., Keshet, U., \& Lemoine, M., 2015, Space Sci. Rev., 191, 519

Medvedev, M. V. \& Zakutnyaya, O. V., 2009, ApJ, 696, 2269

Milosavljevic, M. \& Nakar, E., 2006, ApJ, 651, 979

Derishev, E. \& Piran, T. 2016, MNRAS, 460, 2036

Arber, T. D., Bennett, K., Brady, C. S., Lawrence-Douglas, A., Ramsay, M. G., Sircombe, N. J., Gillies, P., Evans, R. G., Schmitz, H., Bell, A. R., \& Ridgers, C. P., 2015, Plasma Physics and Controlled Fusion, 57, 113001

Garasev, M. \& Derishev, E. 2016, MNRAS, 461 (1), 641 